\title{
Tapered Optical Fiber Coated with ZnO Nanorods for Detection of Ethanol Concentration in Water
}

\author{
Saeed Azad, Roghaieh Parvizi*, and Ebrahim Sadeghi \\ Department of Physics, University of Yasouj, City Yasouj, Country Iran \\ *Corresponding author Email: parvizi.r@yu.ac.ir
}

Received: Jul. 2, 2017, Revised: Jul. 31, 2017, Accepted: Sep. 14, 2017, Available Online: Dec. 1, 2018

DOI: 10.29252/ijop.12.2.109

\begin{abstract}
This work presents $\mathrm{ZnO}$ nanorods coated multimode optical fiber sensing behavior in response to ethanol solution. The sensor operates based on modulation of light intensity which arises from manipulation of light interaction with the ambient environment in sensing region. For this purpose, two steps are experimentally applied here; etching and then coating fiber with $\mathrm{ZnO}$ nanorods to provide stronger evanescent waves causing an enhanced interaction. Long length of fiber $(15 \mathrm{~mm})$ was etched uniformly and then well-ordered $\mathrm{ZnO}$ nanorods were grown hydrothermally on the core of optical fiber. Fiber coated with $\mathrm{ZnO}$ demonstrated an enhanced sensing performances such that response time decreased to $0.6 \mathrm{~s}$, linearity increased to $97 \%$ and sensitivity improved. Applicable features of the proposed device such as fast response time and high linearity make it favorable candidate for fiber optic sensing applications.
\end{abstract}

KEYWORDS: Evanescent field, Ethanol sensor, Hydrothermal, Optical fiber sensor, $\mathrm{ZnO}$ nanorods.

\section{I.INTRODUCTION}

Nowadays real-time and in-situ detection of ethanol with high sensitivity is of great importance in many industrial and biological applications. Among different sensing approaches, the efficient technique in determination of alcohols is based on optical devices. Optical sensors possess inherent advantages of safety, miniaturization and costeffective for demanding analytical applications. A number of ethanol sensors have reported based on Fabry-Perot cavity [1], fluorescence [2-6], Raman spectroscopy [7] and optical fibers [5, 8-12]. Owing to their intrinsic advantages, fiber optic sensors are an ideal candidate and have been employed for ethanol sensing [8, 13, and 14]. In this regard, Penza et al. [10] deposited single-walled carbon nanotube on optical fiber through Langmuir-Blodgett technique for ethanol sensing application at room temperature. In another study, Zhengtian et al. [12] investigated response of long period fiber coated with $\mathrm{SnO} 2$ thin film to ethanol. Fiber optic sensor overlaid with metal oxide nanostructures ( $\mathrm{TiO} 2, \mathrm{ZnO}$ and $\mathrm{SnO} 2$ ) exhibited enhancement in sensor performances due to high surface to volume ratio and applicable chemical and physical properties $[11$, 15-18]. More specifically, $\mathrm{ZnO}$ nanostructures with a direct wide band gap around of $3.2 \mathrm{eV}$ at room temperature, reactive surfaces for chemisorption $[19,20]$ and large exciton binding energy $(\sim 60 \mathrm{meV})$ render it as a suitable choice for sensor fabrication. Among various morphologies of $\mathrm{ZnO}$, onedimensional structure is of exclusive feature of high surface-to-volume ratio and is relatively simple to grow in aqueous solutions due to the polar nature of the (002) crystal plane [21]. Different methods have been devoted to synthesize $\mathrm{ZnO}$ nanorods, including physical and chemical vapor deposition [22, 23] and vapor transport [24]. These methods require a relatively high temperature and complex vacuum environment during the growth process. On the other hand, hydrothermal method of growing $\mathrm{ZnO}$ nanorods is simple, environmentally friendly and energy-efficient $[25,26]$. In recent decades, hydrothermally 
growing nanorods on the flat substrates have been studied intensively [27-30]. However, few published works in the literature investigated the well-arrayed dense nanorods on the curved surfaces such as optical fiber $[15,31]$. In evanescent wave based fiber optic sensors a certain ratio of the transmitted light through the fiber is leaked out by removing a certain region of the cladding of the fiber which is known as evanescent waves. In the modified cladding region, the evanescent wave trapped through the coated nanostructure provides the interaction with the external medium. This technique was exploited for developing evanescent wave absorption based sensors. Absorption of the evanescent field occurs when the nanostructure medium, which forms and modifies the cladding of the waveguide, enable to interact with the analyte [32-35]. Herein variation of refractive index corresponding to different concentration of analyte will affect the absorption of evanescent wave in the sensing region. Since $\mathrm{ZnO}$ coated optical fiber is able to interact with the ethanol solution giving rise to the manipulation of output intensity and providing a highly sensitive sensor.

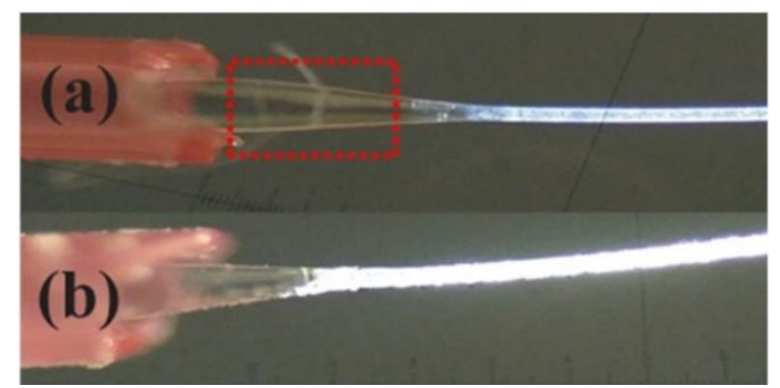

Fig. 1. Microscope image of (a) bare optical fiber etched to $28 \mu \mathrm{m}$ in diameter, (b) $\mathrm{ZnO}$ coated fiber with same diameter.

Presence of nanostructures with higher refractive index than optical fiber provides more evanescent waves on the sensing region that improve and facilitate light interaction with ambient medium. In the present paper, large-scale arrays of highly ordered $\mathrm{ZnO}$ nanorods were grown hydrothermally on the etched multimode silica fiber. The behavior of thinned bare fiber was compared with $\mathrm{ZnO}$ coated fiber in terms of evanescent wave generation and sensing performances. Modes distribution in fiber core under different refractive index around $\mathrm{ZnO}$ coated fiber was also studied theoretically.

\section{EXPERIMENTS}

\section{A. Sensing Principle and Sensor Setup}

The proposed sensor operation is fundamentally tied to the variation of the fiber surrounding environment refractive index. By changing the concentration of gas or liquid, the refractive index around sensing region would change which leads to variation in output light intensity. Since these fashions of sensors work in the principle of evanescent waves, the key point for designing high sensitive sensor with fast response is providing high evanescent field in sensing region [36]. Thinner fiber resulted in a stronger interaction for sensing applications; more evanescent wave can be produced by higher light leakage in tiny fibers. In this regard different methods have been reported including tapering fiber through flame heated method [15], mechanically removing clad of fiber [37, 38] and chemical etching [39]. In this work, long length of fiber thinned with high smoothness through low cost chemical etching method. Referring to our recent experiment [36] the efficient evanescent waves attained for multimode fiber with 28 $\mu \mathrm{m}$ in diameter, near $76 \%$ of coupled light leaked out the fiber core. It should be noted that utilizing nanostructure with higher refractive index than fiber core allows higher light leakage. As shown in Fig. 1 the effect of etching fiber and growth of nanorods in generation of evanescent field is demonstrated experimentally. Figures 1(a) and 1(b) illustrate evanescent field, the emerged light around the fiber, for the bare and the $\mathrm{ZnO}$ coated fiber, respectively. It is clear that in the beginning of cone shaped region (as shown in dotted rectangular in Fig. 1(a) light didn't leak out, while in the thinner region it is able to leaked out as evanescent fields. $\mathrm{ZnO}$ coated fiber allowing higher portion of light leaked out of the fiber core and propagated in modified clad $(\mathrm{ZnO})$ region. 


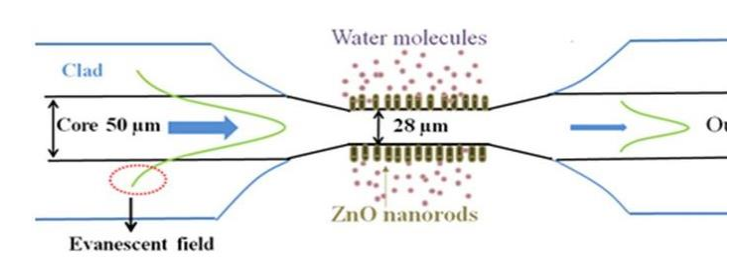

(b)

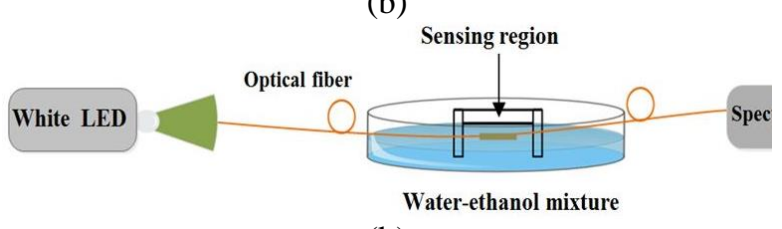

(b)

Fig. 2. (a) Sensing region (b) Experimental setup in ambient conditions for real-time monitoring of transmitted light intensity through optical fiber during alcohol sensing.

This phenomenon provides more interaction of light with external stimulus. $\mathrm{ZnO}(\mathrm{n} \sim 1.9)$ with higher refractive index than silica core ( $\mathrm{n}$ $\sim 1.45$ ) operates such as waveguide, moreover it can chemisorb and physisorb different kind of analyte applied as a suitable candidate for sensing applications.

Figure 2 shows the experimental setup of optical fiber sensor that immersed in waterethanol mixture with different concentration. It consists of LED light source (model: HNKHP003-E45) with wavelength emission ranging from $400-800 \mathrm{~nm}$ coupled to the multimode fiber. It should be noted that according to our previous experiment [40], maximum intensity variation occurs at wavelength of $560 \mathrm{~nm}$ so the rest of sensor measurements and calculations were done at this particular wavelength. The light emerging from the other end of the fiber is fed to a spectrometer (model: HR 4000 CG-UV-NIR). The signal from spectrometer was transferred to computer and output intensity corresponds to each concentration was recorded. It should be noted that real-time monitoring of transmitted light intensity inside optical fiber was done through high resolution spectrophotometer to precisely obtaining sensor time responses. Once sensing region immersed in solution, air around the fiber replaced with water-alcohol molecules, two different responses observed for bare and $\mathrm{ZnO}$ coated fiber. In the former, noticeable increase in output intensity was observed. Hydrophilic property of silica fiber core leads to back reflection of leaked light into the core causing increase in output intensity [41]. By increasing alcohol concentration and so increasing surrounding refractive index than that of pure water, output was decreased in consistence with theory and higher portion of light leaked out. For the latter case, water molecules chemisorbed on the $\mathrm{ZnO}$ nanorods surface and there was no more contact between fiber and water molecules so by increasing alcohol concentration output light intensity decreased.

\section{B. Sensing Region Preparation}

Sensing region prepared through chemically removing the cladding of multimode fiber (core/cladding diameters of 50/125 $\mu \mathrm{m}$ and numerical aperture of $0.2 \pm 0.015$ ) up to the core. Middle segment of fiber, with $15 \mathrm{~mm}$ length, immersed in the diluted $25 \%$ HF (HF $40 \%$, E. Merck) acid solution, such that the average etching rate for cladding removal was about $0.75 \mu \mathrm{m} / \mathrm{min}$ and it was about 1.16 $\mu \mathrm{m} / \mathrm{min}$ to decrease fiber core diameter. Fiber waist diameter etched to $28 \mu \mathrm{m}$ and then rinsed with deionized water. $\mathrm{ZnO}$ seed particles were uniformly covered silica fiber as a substrate by dipping the fiber into the seeding solution [36] for $10 \mathrm{~min}$ and then annealed at $150{ }^{\circ} \mathrm{C}$ in the oven for $20 \mathrm{~min}$ to evaporate the solvent, this process repeated three times. $\mathrm{ZnO}$ nanorods obtained after hydrothermal treatment in autoclave at $95^{\circ} \mathrm{C}$ for 5 hours. Equimolar aqueous growth solution $(0.01 \mathrm{M})$ of zinc nitrate hexahydrate ( $\mathrm{Zn}$ (NO3).6H2O, 99\% purity, E. Merck) and hexamine (HMTA, 99\% purity, E. Merck) (C6H12N4) dissolved in $100 \mathrm{ml}$ deionized water and poured in Teflon-lined sealed autoclave. Finally, the fibers were taken out of the growth solution and rinsed through deionized water carefully followed by baking at $150{ }^{\circ} \mathrm{C}$ for 1 hour.

\section{RESULTS AND DISCUSSION}

\section{A. SEM Analysis}

Figure $3(\mathrm{a}, \mathrm{b})$ illustrate SEM images of the bare optical fiber and seeded fiber covered with $\mathrm{ZnO}$ nanorods. Bare fiber etched uniformly with long-length, providing applicable substrate for vertical growth of 
nanorods and sensing applications. Over laid fiber exhibit well-arrayed and relatively high density of $\mathrm{ZnO}$ nanorods pointed outwards from the fiber surface with aspect ratio more than 8 and average length/diameter of 2 $\mu \mathrm{m} / 220 \mathrm{~nm}$. Fibers seeding with $\mathrm{ZnO}$ nanoparticles establish nucleation sites and lowering the thermodynamic barrier resulting improvement in aspect ratio and density [42]. Employing equimolar solution of zinc nitrate and hexamine in hydrothermal treatment causes growth of $\mathrm{ZnO}$ rods with hexagonal wurtzite structure [42].

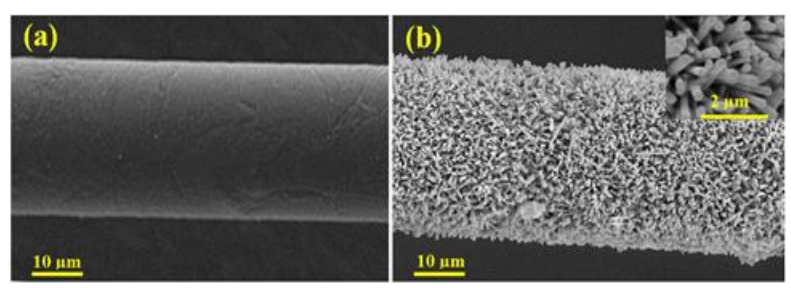

Fig. 3. SEM images of (a) etched optical fiber and (b) Seeded fiber covered with $\mathrm{ZnO}$ nanorods (inset shows enlarge view).

\section{B. PL Analysis}

PL analysis is a powerful study that exhibit the absorption of energy and subsequent emission of light under the term luminescence providing noticeable knowledge related to the material defects originated during growth process. Figure 4 shows the room temperature photoluminescence (PL) spectra obtained from the silica optical fiber covered by $\mathrm{ZnO}$ nanorods. Since coated optical fiber was too thin, to better observe the emitted luminescence several identical coated fibers put in the sample holder and excited at wavelengths of $240 \mathrm{~nm}$. It can be seen that samples show a typical luminescence behavior with the two emissions of a strong UV peak around $397 \mathrm{~nm}$ and a broad deep level peak with green emission in the 490 560 nm region. The UV emission is due to recombination of free-excitons through exciton-exciton collision process [43]. It has been suggested that green band emission is related to singly ionized oxygen vacancy in $\mathrm{ZnO}[44,45]$, resulting from recombination of a photo-generated hole with the singly ionized charge [46]. Oxygen vacancies and zinc interstitials, point defects, are critical characteristic in sensing applications as they cause very large variation in the surface conductivity [47]. Changes in charge density have direct impact on optical properties, namely refractive index of nanorods altered correspondingly [48].

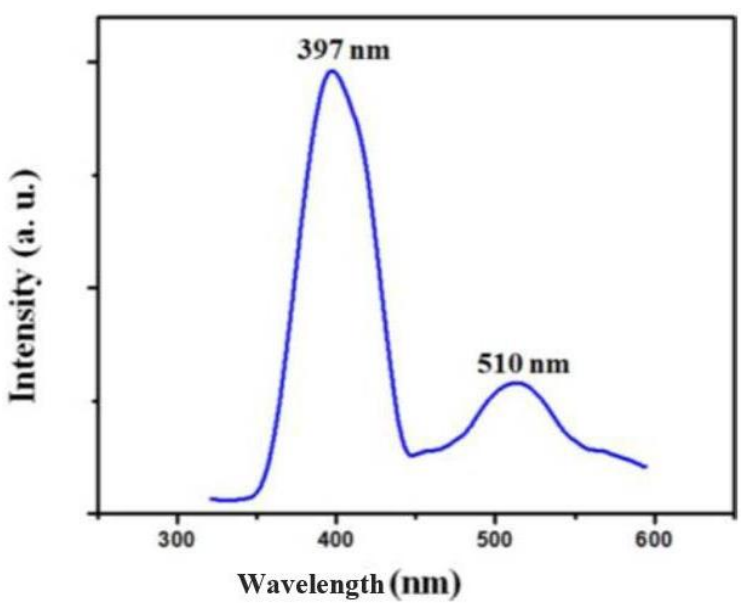

Fig. 4. Room-temperature PL spectrum of $\mathrm{ZnO}$ nanorods that are grown on the optical fiber excited at wavelength of $240 \mathrm{~nm}$.

\section{Sensor Response to Ethanol}

To investigate sensor response to different ethanol concentration, sensing region was immersed in ethanol-water mixture for about 2 min and was withdrawn out and stayed in air for a few seconds this process repeated consecutively for different concentrations. Range of ethanol concentration in distilled water was from 0-80 volume percent. First, fibers immersed in pure water, as mentioned above bare fiber exhibited different behavior compare to $\mathrm{ZnO}$ coated fiber. The output intensity plotted as a function of ethanol volume percentage (Fig.5 (a, and b)). Transmitted light intensity reach to its stable value after $0.8 \mathrm{~s}$ for bare fiber and $0.6 \mathrm{~s}$ for $\mathrm{ZnO}$ coated fiber as immersed in solution.

When the fiber was withdrawn out from solution, spiky signal appeared and proceed towards the initial value (that of in air). Since residual of solution's droplets exist on the fiber surface, the transmitted light couldn't return to its initial value. Herein, we tried to exhibit sensors ability to consecutive measurements and its distinction accuracy for solution with different concentration. 

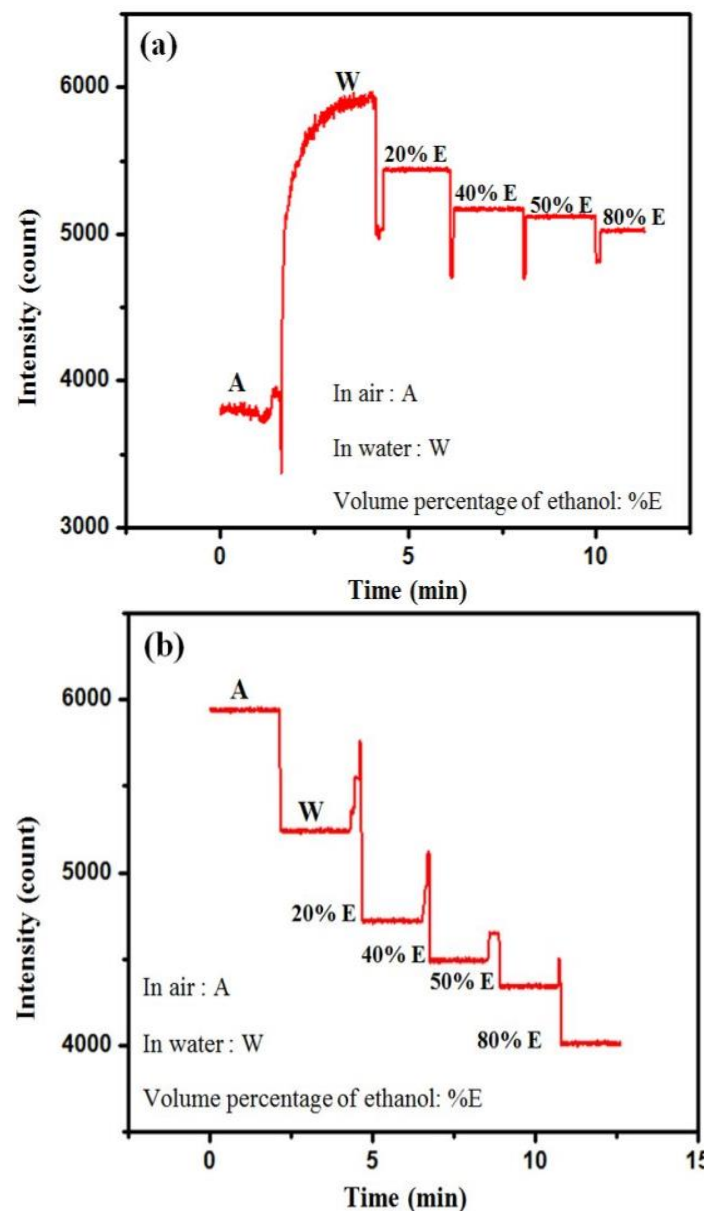

Fig. 5. Change in output intensity upon exposure to different ethanol concentration versus exposure time for (a) bare fiber and (b) $\mathrm{ZnO}$ coated fiber.

As mentioned above, bare and $\mathrm{ZnO}$ coated fibers immersed in ethanol solution with different concentration. In order to investigate the sensitivity and linearity of the sensor response, the output light intensity against ethanol concentration of was considered as illustrated in Fig. 6. The output intensity decreased by increasing ethanol concentration. It can be seen that sensitivity (defined as slope of fitted line) enhanced from -10.69 (count $/ \%$ ) for bare fiber to -14.94 (count/\%) for $\mathrm{ZnO}$ coated fiber. Sensor linearity improved from $87 \%$ for bare fiber to $97 \%$ for $\mathrm{ZnO}$ coated fiber. Minus sign in sensitivity refers to decreasing in transmitted light intensity by increasing in ethanol concentration. These intensity variations in tapered optical fiber are described by the lambert-Beer Law [17, 49] as shown by the following equation:

$I=I_{0} e^{-\gamma l}, \gamma=\alpha_{m} r_{f} C$ where $I$ and $I_{0}$ are the light intensities before $\left(I_{0}\right)$ and after $(I)$ interaction of sensing region with analyte. The values $C$ and $l$ are the concentration of analyte and length of tapered fiber, respectively. The parameter $\gamma$ stands for evanescent wave absorption coefficient, which is the function of concentration, effective fraction of the total guided power $\left(r_{f}\right)$ in the sensing region and $\alpha_{m}$ that is the bulk absorption coefficient of the absorbing material.

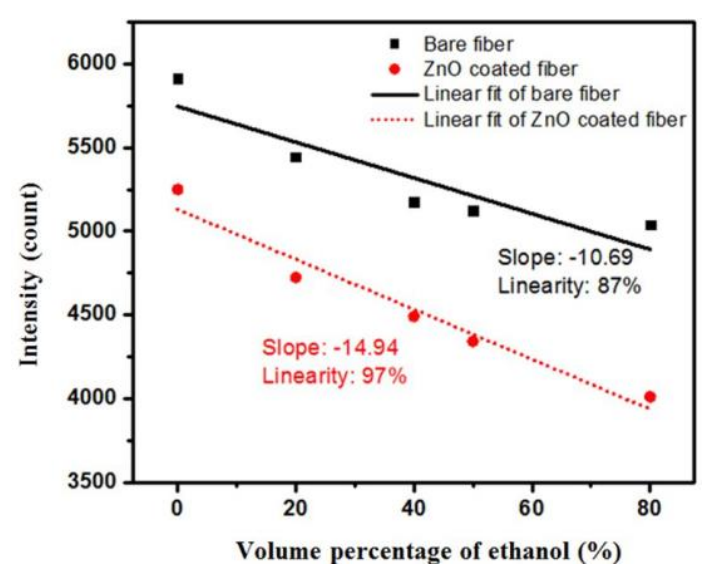

Fig. 6. Output intensity variation against ethanol concentration.

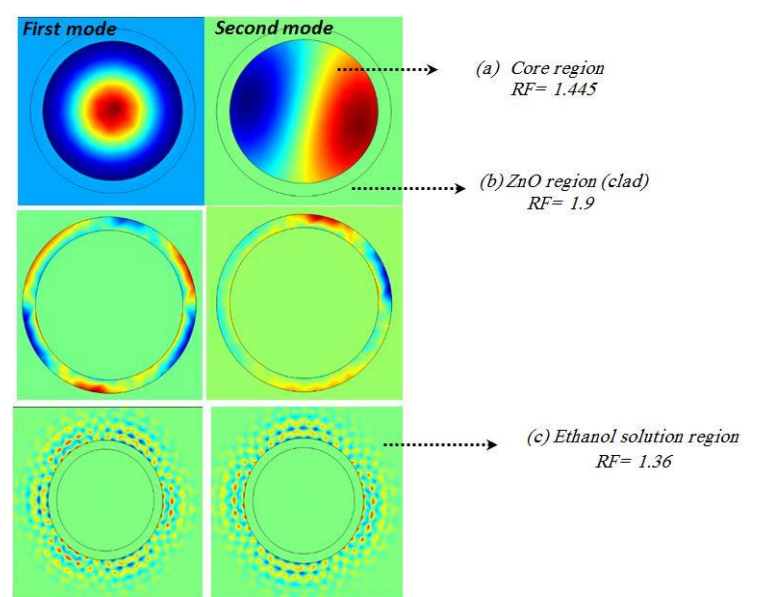

Fig. 8. Numerical simulation of light modes distribution in: (a) core, (b) clad, (c) ethanol solution regions.

In order to investigate light scattering through $\mathrm{ZnO}$ nanorods, which is practically illustrated in Fig. 1, we have simulated light modes distribution in the core, clad and solution regions. The optical properties of fiber were studied through a two-dimensional finite 
element method. The information about fiber structure used for simulation is as follows. The diameter of fiber core is $28 \mu \mathrm{m}$. The refractive index of the fiber core and $\mathrm{ZnO}$ layer (thin ring with $2 \mu \mathrm{m}$ thickness around the core, defining clad) were considered 1.445 and 1.9, respectively. The refractive index related to different concentration of ethanol was measured experimentally through Abbe optical refractometer and was plotted in Fig. 7.

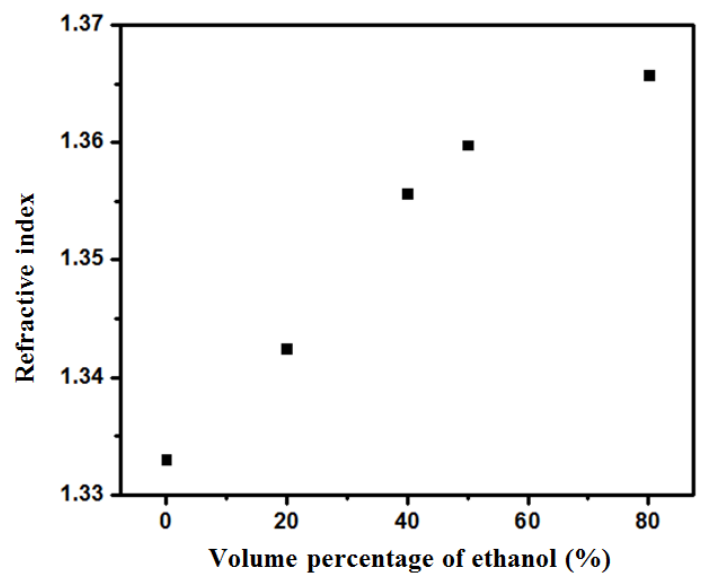

Fig. 7. Refractive index of different concentration of ethanol measured through Abbe refractometer.

The measured refractive index of the solution was varied from about 1.33 to 1.37 against 0 to 80 volume percentage of ethanol in distilled water. Accordingly, these measured refractive indices can be treated as the initial refractive index values for defining the solution media in the simulation. The two fundamental modes in different regions were shown in Fig. 8. It is clearly seen that the light leaks out near fiber surface and penetrating to ambient medium giving rise to the reduction of transmitted output intensity.

\section{CONCLUSION}

In summary, the effect of etching fiber and growth of nanorods in the magnitude of generated evanescent field was demonstrated experimentally. Well-ordered and relatively high density of $\mathrm{ZnO}$ nanorods were grown successfully on the fiber with aspect ratio more than 8. Employing equimolar solution of zinc nitrate and hexamine in hydrothermal treatment resulted $\mathrm{ZnO}$ nanorods with hexagonal wurtzite structure. PL analysis indicated presence of oxygen vacancy on the surface of grown nanorods that are dominant point defects and play an important role in sensing process, inducing conductivity variations and resulting refractive index changes. Sensing behavior of the bare fiber compared to $\mathrm{ZnO}$ coated fiber in response to ethanol solution (10-80\% volume percentage). Results revealed that $\mathrm{ZnO}$ coated fiber enhanced sensor performances, time response was measured near $0.6 \mathrm{~s}$, sensitivity more than 14.9 (count/\%) and high linearity of 97\% exhibited proposed structure is attractive for chemical and biological applications. To investigate more precisely the light leakage and light modes distribution under different concentration of ethanol numerical simulation was carried out.

\section{ACKNOWLEDGMENT}

The authors express their appreciation to the Research Council of the University of Yasouj for financial support of this work.

\section{REFERENCES}

[1] J. Gao, T. Gao, and M. J. Sailor, "Poroussilicon vapor sensor based on laser interferometry," Appl. Phys. Lett. vol. 77, no. 6, pp. 901-903, 2000.

[2] H. Tang, Y. Li, C. Zheng, J. Ye, X. Hou, and Y. Lv, "An ethanol sensor based on cataluminescence on $\mathrm{ZnO}$ nanoparticles," Talanta, vol. 72, no. 4, pp. 1593-1597, 2007.

[3] D. Simon, R. Czolk, and H. Ache, "Doped solgel films for the development of optochemical ethanol sensors," Thin Solid Films, vol. 260, no. 1, pp. 107-110, 1995.

[4] S. Petrova, Y. Kostov, K. Jeffris, and G. Rao, "Optical ratiometric sensor for alcohol measurements," Analytical Lett. vol. 40, no. 4, pp. 715-727, 2007.

[5] G. Orellana, A. M. Gomez-Carneros, C. de Dios, A. A. Garcia-Martinez, and M. C. Moreno-Bondi, "Reversible fiber-optic fluorosensing of lower alcohols," Anal. Chem. vol. 67 , no. 13 , pp. 2231-2238, 1995.

[6] P. Blum, G. J. Mohr, K. Matern, J. Reichert, and U. E. Spichiger-Keller, "Optical alcohol sensor using lipophilic Reichardt's dyes in 
polymer membranes," Anal. Chem. Acta, vol. 432, no. 2, pp. 269-275, 2001.

[7] D. Cleveland, M. Carlson, E. D. Hudspeth, L. E. Quattrochi, K. L. Batchler, S. A. Balram, S. Hong, and R. G. Michel, "Raman spectroscopy for the undergraduate teaching laboratory: Quantification of ethanol concentration in consumer alcoholic beverages and qualitative identification of marine diesels using a miniature Raman spectrometer," Spectrosc. Lett. vol. 40, no. 6, pp. 903-924, 2007.

[8] Y. Kurauchi, T. Yanai, N. Egashira, and K. Ohga, "Fiber-Optic Sensor with a Chitosan/Poly (vinyl alcohol) Cladding for the Determination of Ethanol in Alcoholic Beverages," Anal. Sci. vol. 10, no. 1, pp. 213217, 1994.

[9] M. Penza, G. Cassano, P. Aversa, F. Antolini, A. Cusano, A. Cutolo, M. Giordano, and L. Nicolais, "Alcohol detection using carbon nanotubes acoustic and optical sensors," Appl. Phys. Lett. vol. 85, no. 12, pp. 2379-2381, 2004.

[10] M. Penza, G. Cassano, P. Aversa, A. Cusano, A. Cutolo, M. Giordano, and L. Nicolais, "Carbon nanotube acoustic and optical sensors for volatile organic compound detection," Nanotechnol. vol. 16, no. 11, pp. 2536-2547, 2005.

[11]Z. Gu, and $\mathrm{Y} . \mathrm{Xu}$, "Design optimization of a long-period fiber grating with sol-gel coating for a gas sensor," Meas. Sci. Technol. vol. 18, no. 11, pp. 3530-3536, 2007.

[12]Z. Gu, Y. Xu, and K. Gao, "Optical fiber longperiod grating with solgel coating for gas sensor," Opt. Lett. vol. 31, no. 16, pp. 24052407, 2006.

[13]C. Elosua, C. Bariain, I. R. Matias, F. J. Arregui, E. Vergara, and M. Laguna, "Optical fiber sensing devices based on organic vapor indicators towards sensor array implementation," Sens. Actuator B-Chem. vol. 137, no. 1, pp. 139-146, 2009.

[14] G. Possetti, L. Côcco, C. Yamamoto, L. De Arruda, R. Falate, M. Muller, and J. Fabris, "Application of a long-period fibre gratingbased transducer in the fuel industry," Meas. Sci. Technol. vol. 20, no. 3, pp. 034012-9, 2009.
[15] Y. Liu, Y. Zhang, H. Lei, J. Song, H. Chen, and B. Li, "Growth of well-arrayed $\mathrm{ZnO}$ nanorods on thinned silica fiber and application for humidity sensing," Opt. Express, vol. 20, no. 17, pp. 19404-19411, 2012.

[16] M. Batumalay, Z. Harith, H. Rafaie, F. Ahmad, M. Khasanah, S. Harun, R. Nor, and H. Ahmad, "Tapered plastic optical fiber coated with $\mathrm{ZnO}$ nanostructures for the measurement of uric acid concentrations and changes in relative humidity," Sens. Actuator B-Chem. vol. 210, pp. 190-196, 2014.

[17] A. Urrutia, J. Goicoechea, and F. J. Arregui, "Optical fiber sensors based on nanoparticleembedded coatings," Sensors, vol. 2015, pp. 805053 (1-18), 2015.

[18]Z. L. Wang, "Oxide nanobelts and nanowires-growth, properties and applications," J. Nanosci. Nanotechnol. vol. 8, no. 1, pp. 27-55, 2008.

[19]D. C. Look, "Recent advances in $\mathrm{ZnO}$ materials and devices," Mater. Sci. Eng. B, vol. 80, no. 1, pp. 383-387, 2001.

[20] Ü. Özgür, Y. I. Alivov, C. Liu, A. Teke, M. Reshchikov, S. Doğan, V. Avrutin, S.-J. Cho, and H. Morkoc, "A comprehensive review of $\mathrm{ZnO}$ materials and devices," J. Appl. Phys. vol. 98, no. 4, pp. 041301-103, 2005.

[21]A.K. Singh, Advanced x-ray techniques in research and industry, IOS Press, 2005.

[22] C. Gorla, N. Emanetoglu, S. Liang, W. Mayo, Y. Lu, M. Wraback, and H. Shen, "Structural, optical, and surface acoustic wave properties of epitaxial $\mathrm{ZnO}$ films grown on (0112) sapphire by metalorganic chemical vapor deposition," J. Appl. Phys. vol. 85, no. 5, pp. 2595-2602, 1999.

[23] Y. Kong, D. Yu, B. Zhang, W. Fang, and S. Feng, "Ultraviolet-emitting $\mathrm{ZnO}$ nanowires synthesized by a physical vapor deposition approach," Appl. Phys. Lett. vol. 78, no. 4, pp. 407-409, 2001.

[24] M. H. Huang, Y. Wu, H. Feick, N. Tran, E. Weber, and P. Yang, "Catalytic growth of zinc oxide nanowires by vapor transport," Adv. Mater. vol. 13, no. 2, pp. 113-116, 2001.

[25] A. O. Dikovska, P. Atanasov, A. T. Andreev, B. Zafirova, E. Karakoleva, and T. Stoyanchov, "ZnO thin film on side polished optical fiber for gas sensing applications," 
Appl. Surf. Sci. vol. 254, no. 4, pp. 10871090, 2007.

[26] S. Baruah and J. Dutta, "Hydrothermal growth of $\mathrm{ZnO}$ nanostructures," Sci. Technol. Adv. Mater. vol. 10, pp. 013001 (1-18), 2016.

[27] J.-H. Lee, C. Leu, Y.-W. Chung, and M.-H. Hon, "Fabrication of ordered $\mathrm{ZnO}$ hierarchical structures controlled via surface charge in the electrophoretic deposition process," Nanotechnol. vol. 17, no. 17, pp. 4445-4450, 2006.

[28] A. Umar, C. Ribeiro, A. Al-Hajry, Y. Masuda, and Y. Hahn, "Growth of highly c-axisoriented $\mathrm{ZnO}$ nanorods on $\mathrm{ZnO} /$ glass substrate: growth mechanism, structural, and optical properties," J. Phys. Chem. C, vol. 113, no. 33, pp. 14715-14720, 2009.

[29]P. Pant, J. D. Budai, R. Aggarwal, R. Narayan, and J. Narayan, "Structural characterization of two-step growth of epitaxial $\mathrm{ZnO}$ films on sapphire substrates at low temperatures," J. Phys. D, vol. 42, no. 10, pp. 105409 (1-8), 2009.

[30] A. Umar, B.-K. Kim, J.-J. Kim, and Y. Hahn, "Optical and electrical properties of $\mathrm{ZnO}$ nanowires grown on aluminium foil by noncatalytic thermal evaporation," Nanotechnol. vol. 18, no. 17, pp. 175606 (1-7), 2007.

[31]H. Fallah, S. W. Harun, W. S. Mohammed, and J. Dutta, "Excitation of core modes through side coupling to multimode optical fiber by hydrothermal growth of $\mathrm{ZnO}$ nanorods for wide angle optical reception," J. Opt. Soc. Am. B, vol. 31, no. 9, pp. 22322238, 2014.

[32]B. G. S. KHijwania, "Experimental studies on the response of the fiber optic evanescent field absorption sensor," Fiber Integ. Opt. vol. 17, no. 1, pp. 63-73, 1998.

[33] J. Luo, J. Yao, Y. Lu, W. Ma, and X. Zhuang, "A silver nanoparticle-modified evanescent field optical fiber sensor for methylene blue detection," Sensors, vol. 13, no. 3, pp. 39863997, 2013.

[34]D. J. Sirbuly, A. Tao, M. Law, R. Fan, and P. Yang, "Multifunctional nanowire evanescent wave optical sensors," Adv. Mater. vol. 19, no. 1, pp. 61-66, 2007.

[35]S. Shukla, M. Rani, N. K. Sharma, and V. Sajal, "Sensitivity enhancement of a surface plasmon resonance based fiber optic sensor utilizing platinum layer," Optik, vol. 126, no. 23, pp. 4636-4639, 2015.

[36] S. Azad, E. Sadeghi, R. Parvizi, A. Mazaheri, and M. Yousefi, "Sensitivity optimization of $\mathrm{ZnO}$ clad-modified optical fiber humidity sensor by means of tuning the optical fiber waist diameter," Opt. Laser Technol. vol. 90, pp. 96-101, 2017.

[37] S. Manivannan, A. Saranya, B. Renganathan, D. Sastikumar, G. Gobi, and K. C. Park, "Single-walled carbon nanotubes wrapped poly-methyl methacrylate fiber optic sensor for ammonia, ethanol and methanol vapors at room temperature," Sens. Actuator B-Chem. vol. 171, pp. 634-638, 2012.

[38]G. Liu and D. Feng, "Evanescent wave analysis and experimental realization of refractive index sensor based on D-shaped plastic optical fiber," Optik, vol. 127, no. 2, pp. 690-693, 2016.

[39]H. J. Kbashi, "Fabrication of submicrondiameter and taper fibers using chemical etching," J. Mater. Sci. Technol. vol. 28, no. 4, pp. 308-312, 2012.

[40]S. Azad, E. Sadeghi, R. Parvizi, and A. Mazaheri, "Fast response relative humidity clad-modified multimode optical fiber sensor with hydrothermally dimension controlled ZnO nanorods," Mat. Sci. in Semicon. Proc, vol. 66, pp. 200-206, 2017.

[41]M. Ogita, Y. Nagai, M. Mehta, and T. Fujinami, "Application of the adsorption effect of optical fibres for the determination of critical micelle concentration," Sens. Actuator B-Chem. vol. 64, no. 1, pp. 147-151, 2000.

[42] L. Vayssieres, K. Keis, S.-E. Lindquist, and A. Hagfeldt, "Purpose-built anisotropic metal oxide material: 3D highly oriented microrod array of ZnO," J. Phys. Chem. B, vol. 105, no. 17, pp. 3350-3352, 2001.

[43]L. Dai, X. Chen, W. Wang, T. Zhou, and B. $\mathrm{Hu}$ "Growth and luminescence characterization of large-scale zinc oxide nanowires," J. Phys. Condens. Matter, vol. 15, no. 13 , pp. 2221-2226, 2003.

[44] Q. Ahsanulhaq, J. Kim, N. Reddy, and Y. Hahn, "Growth mechanism and characterization of rose-like microspheres and hexagonal microdisks of $\mathrm{ZnO}$ grown by surfactant-free solution method," J Ind. Eng. Chem. vol. 14, no. 5, pp. 578-583, 2008. 
[45]J. Hu and Y. Bando, "Growth and optical properties of single-crystal tubular $\mathrm{ZnO}$ whiskers," Appl. Phys. Lett. vol. 82, no. 9, pp. 1401-1403, 2003.

[46]S. Mridha and D. Basak, "Effect of concentration of hexamethylene tetramine on the structural morphology and optical properties of $\mathrm{ZnO}$ microrods grown by low-temperature solution approach," Phys. Status Solidi A, vol. 206, no. 7, pp. 15151519, 2009.

[47]A. Kumar, "Performance analysis of Zinc oxide based alcohol sensors," Int. J. Appl. Sci. Eng. Res. vol. 4, no. 4, pp. 427-436, 2015.

[48] M. Konstantaki, A. Klini, D. Anglos, and S. Pissadakis, "An ethanol vapor detection probe based on a $\mathrm{ZnO}$ nanorod coated optical fiber long period grating," Opt. Express, vol. 20, no. 8, pp. 8472-8484, 2012.

[49] S. H. Girei, A. A. Shabaneh, H. Ngee-Lim, M. N. Hamidon, M. A. Mahdi, and M. H. Yaacob, "Tapered optical fiber coated with graphene based nanomaterials for measurement of ethanol concentrations in water," Opt. Rev. vol. 22, no. 3, pp. 385-392, 2015.

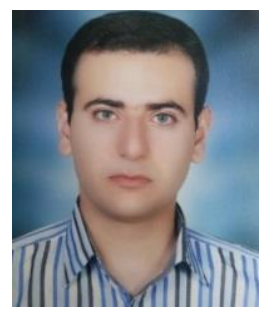

Saeed Azad received the B.Sc. degree in atomic and molecular Physics from Shahid Bahonar University, Kerman, Iran, in 2009 and M.Sc. degree in solid state physics from Bu-Ali Sina University, Hamadan, Iran, in 2011, and Ph.D. degrees in condensed matter physics from University of Yasouj in 2017. Currently, he is a research assistance \& AFM promoter in University of Yasouj, Iran. His research interests include optoelectronics, synthesizing nanostructures and optical sensor based on the growth of nanostructures on the different type of optical fiber.

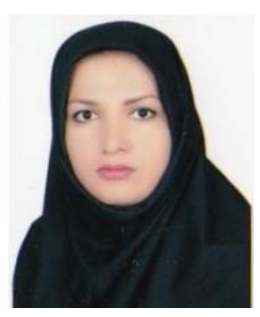

Roghaieh Parvizi earned bachelor degree in Physics at Yasouj University. Later, she graduated from International Center for Science, High Technology, and Environmental Sciences (ICST), Kerman, Iran, to earn her master degree in Optics and Laser. She was honored to graduate her Ph.D in Fiber optic technology (optoelectronics) at University of Malaya with distinction award. Now, she is employed as a lecturer in Yasouj University. Her publications are about optical fiber amplifier, nonlinear based fiber laser generation, quantum dot field theory and optical sensors. Her awards can be listed as follow: Bright Spark Unit member in University of Malaya for one year (20112012), Research Assistance (RA) in University of Malaya for two years (2011 and 2012), Distinction award in Ph.D. degree and distinction award in M.Sc. degree. Currently, she is lecturer at Faculty of Physics, University of Yasouj, Iran.

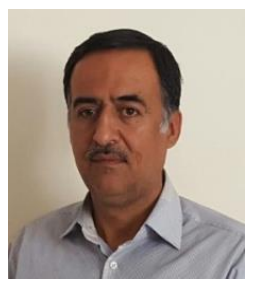

Ebrahim Sadeghi received the B.Sc. degree in Physics from Shiraz University, Iran in 1991, and M.Sc. and Ph.D. degrees in solid state physics from University of Shiraz in 1994 and 2004, respectively. Currently, he is full professor at Faculty of Physics, University of Yasouj, Iran. His research interests include study the optical and electrical properties of quantum wells, wires and dots and optical fiber sensors. 
THIS PAGE IS INTENTIONALLY LEFT BLANK. 